\title{
Homeobox C4 promotes hepatocellular carcinoma progression by the transactivation of Snail
}

\author{
Tao YANG ${ }^{1, *}$, Xian-Bo ZHANG ${ }^{2, *}$, Xiao-Na LI ${ }^{3, *}$, Ming-Ze SUN ${ }^{1}$, Peng-Zhi GAO ${ }^{4}$ \\ ${ }^{1}$ Department of Surgical Oncology, Qingdao Central Hospital, Qingdao, Shandong, China; ${ }^{2}$ Department of Oncology, Hebei General Hospital, \\ Shijiazhuang, Hebei, China, ${ }^{3}$ Department of General Surgery, Qingdao Central Hospital, Qingdao, Shandong, China, ${ }^{4}$ Department of General \\ Surgery, The First Hospital of Shijiazhuang City, Shijiazhuang, Hebei, China
}

*Correspondence: lixiaona1441@163.com

${ }^{*}$ Contributed equally to this work.

Received April 3, 2020 / Accepted July 9, 2020

\begin{abstract}
Homeobox C4 (HOXC4) belongs to the homeoprotein family of transcription factors, which play a critical role in morphogenesis and differentiation during embryonic development. Aberrant expression of HOXC4 has been reported in several types of cancers. However, the role of HOXC4 in hepatocellular carcinoma (HCC) remains unknown. Here, we reported that HOXC4 is upregulated in HCC tissues and predicts a poor outcome in patients with HCC. HOXC4 promotes HCC progression and induces an EMT-like phenotype both in vitro and in vivo. Furthermore, we demonstrated that the EMT-related transcription factor Snail is a transcriptional target of HOXC4 and HOXC4 regulates EMT by regulation of transforming growth factor $\beta$ (TGF- $\beta$ ) signaling in HCC. Together, our study suggests that HOXC4 as a novel potential therapeutic target for HCC therapy.
\end{abstract}

Key words: HOXC4, Snail, TGF- $\beta$, epithelial-mesenchymal transition, hepatocellular carcinoma

Hepatocellular carcinoma (HCC) is one of the common malignant diseases and is the third leading cause of cancerrelated death all around the world [1]. Because of no effective pharmacologic therapies and high metastasis capacity at the early stage, most HCC patients cannot receive curative surgical treatments. In addition, due to its high recurrence and metastasis characteristics, the outcome of HCC even after hepatic resections is still poor. Therefore, understanding the molecular mechanism of the disease is highly desirable for developing novel strategies for the prevention and treatment of HCC.

Homeobox (HOX) genes, a family of at least 200 transcription factors, contain a highly conserved 60 aminoacid homeodomain that binds specifically to DNA [2]. HOX family genes are developmentally regulated genes that play critical roles in tissue patterning [3]. Altered expression of HOX genes clusters (A, B, C, and D) has been observed in many human cancers including prostate cancer, breast cancer, colon cancer, lung cancer, leukemias, and liver cancer $[4,5]$. Increasing evidence showed that elevated expression of HOXC genes correlates with malignancy in various types of cancers [6-10]. HOXC4 induces CD14 expression during the differentiation of acute promyelocytic leukemia cells [11]. Increased expression of HOXC4 is observed in melanoma with distant metastasis and overexpression of HOXC4 promotes the uveal melanoma development and progression $[12,13]$. However, the role of HOXC4 in HCC development and progression remains unknown.

Epithelial to mesenchymal transition (EMT) has been shown to play critical roles in HCC progression. Transforming growth factor- $\beta$ (TGF- $\beta$ ) has been identified as one of the main EMT inducers and proposed as a therapeutic target in HCC $[14,15]$. TGF- $\beta$ binds to TGF- $\beta$ type II receptors, which phosphorylate and activate type I receptors. The activated type I receptors phosphorylate the SMAD2 and SMAD3, which assemble into heteromeric complexes with SMAD4 and then translocate into the nucleus for transcriptional regulation [16]. In addition, Smad-independent signaling pathways have been implicated in TGF- $\beta$-induced EMT, including PI3K-AKT and MAP kinase pathways [17].

In the present study, we investigate the role of HOXC4 in HCC progression. We demonstrated that HOXC4 promotes TGF- $\beta$-induced cancer progression and induces an EMT-like phenotype by transactivation of Snail in HCC. Therefore, our study provides a novel therapeutic target for HCC therapy. 


\section{Materials and methods}

Cell culture and human samples. Human HCC cell lines SMMC-7721, Hep3B, SK-Hep1, HepG2, BEL-7405, and Huh-7 were purchased from the American Type Culture Collection (ATCC) and routinely cultured in Dulbecco's modified Eagle medium (DMEM) (Invitrogen, Carlsbad, CA, USA) supplemented with $10 \%$ fetal bovine serum (FBS, Invitrogen) and $1 \%$ penicillin/streptomycin in a humidified atmosphere of $5 \% \mathrm{CO}_{2}$ at $37^{\circ} \mathrm{C}$.

Specimens were obtained from 102 HCC patients who had undergone surgery for tumor resection at the First Hospital of Shijiazhuang city from February 2003 to December 2008. Tissues were immediately frozen in liquid nitrogen and stored at $-80^{\circ} \mathrm{C}$ until use. Follow-up data were obtained by reviewing the hospital records, direct communication with the patients after hepatic resection. This study was approved by the Institutional Review Board of the First Hospital of Shijiazhuang city and written consent was obtained from all participants.

Plasmids construction and generation of stable cell lines. The full-length HOXC4 cDNA was reverse transcriptase-polymerase chain reaction using total RNA from HepG2 cell line. The primer sequence was: forward primer, 5'-GAATTCATGATCATGAGCTCGTATTT-3'; and reverse primer, 5'-AAGCTTATAACCTGGTAATGTCCT-3'. The PCR product was cloned into EcoR I and Hind III sites of the mammalian expression vector pcDNA3.1 (+) (Invitrogen) (pcDNA3.1-HOXC4). The HOXC4 siRNAs were purchased from Santa-Cruz Biotechnology (siHOXC4). Following the manufacturer's instructions, Huh7 stable transfectants were screened for overexpressed HOXC4 after about three weeks

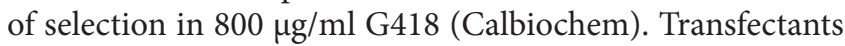
were routinely cultured under selection.

Tissue RNA isolation and reverse transcription quantitative real-time PCR. Total RNA was extracted with TRIZOL reagent according to the manufacturer's instructions. $5 \mu \mathrm{g}$ of total RNA was used to reverse transcribed using SuperScript II and oligo dT following the manufacturer recommendations (Invitrogen). The reverse transcription quantitative real-time PCR (RT-qPCR) analysis was performed using the Fast SYBR Green MasterMix System (Invitrogen) according to the manufacturer's instructions. The PCR conditions were as follows: $95^{\circ} \mathrm{C}$ for $20 \mathrm{~s} ; 95^{\circ} \mathrm{C}$ for $3 \mathrm{~min} ; 62^{\circ} \mathrm{C}$ for $30 \mathrm{~s}$; for 40 cycles by using ABI 7500 (Applied Biosystems). The relative quantification was given by the CT values, determined by triplicate reactions for all of the samples. The triplicate CT values of the detectable gene were averaged, and the CT value of GAPDH was subtracted to obtain $\triangle \mathrm{CT}$. The relative mRNA expression level of target genes was determined as $2^{-\Delta \mathrm{CT}}$.

Western blot. A quantity of $30 \mu \mathrm{g}$ of lysates per sample was separated by SDS-PAGE using $10 \%$ polyacrylamide gels and transferred to PVDF membrane. Membranes were blocked in 5\% skim milk for $1 \mathrm{~h}$ at room temperature, washed, and incubated with primary antibodies HOXC4 (ab230639, 1:1000), Vimentin (ab92547, 1:2000), E-cadherin (ab1416, 1:1000), N-cadherin (ab76011, 1:1000) from Abcam or pSmad2 (\#18338, 1:1000), Twist1 (\#46702, 1:500), Snail (\#3879, 1:500), and Smad2/3 (\#8685, 1:1000) from Cell Signaling Technology overnight at $4{ }^{\circ} \mathrm{C}$. After washed in TBST, membranes were incubated with anti-mouse or antirabbit antibodies (1:3000) at room temperature for $1 \mathrm{~h}$. Protein bands were visualized by the ECL system (Pierce Biotechnology).

Luciferase reporter assays. The fragment of Snail promoter $(-2000$ to +1$)$ was cloned into the pGL3-basic luciferase reporter plasmid (Promega). $5 \times 10^{4}$ HEK-293T cells were seeded in a 12 -well plate and $1 \mu \mathrm{g}$ of luciferase reporter plasmid and pcDNA3.1-HOXC4 were co-transfected with Lipofectamine 3000 (Thermo Fisher Scientific Biotechnology) for $48 \mathrm{~h}$ and $100 \mathrm{ng}$ of Renilla reporter as a normalization control. Firefly and Renilla luciferase activities were determined by a Dual-Luciferase Reporter System (Promega) according to the manufacturer's protocol.

Chromatin immunoprecipitation (ChIP) analysis. ChIP analysis was performed according to the protocol of Upstate Biotechnology. Antibody against HOXC4 was used for immunoprecipitation and normal IgG was used as a negative control. 5\% input DNA was used as a positive control for PCR.

Immunofluorescence. Cells were grown on glass chamber slides fixed with 4\% paraformaldehyde in PBS for $30 \mathrm{~min}$ and penetrated by $0.5 \%$ Triton X-100 for $15 \mathrm{~min}$, then blocked by $3 \%$ BSA for $1 \mathrm{~h}$. After washing with PBS, the cells were incubated with the primary antibody at $4{ }^{\circ} \mathrm{C}$ overnight. After washing with $\mathrm{PBS}$, the cells were then incubated with fluorescein isothiocyanate-conjugated secondary antibody for 1 hour at room temperature and then stained with 4, 6'-diamidino2-phenylindole (DAPI; Invitrogen). The images were visualized with an Olympus microscope (Tokyo).

TGF- $\beta 1$ measurement with ELISA and TGF- $\beta$ signaling assay. Cell culture supernatant was harvested after incubation for $48 \mathrm{~h}$ and centrifuged. The free TGF- $\beta 1$ in the culture medium was measured using a TGF- $\beta 1$ ELISA kit (Invitrogen). Each experiment was performed in five wells and was repeated at least three times. TGF- $\beta$ signaling activity was determined by Cignal SMAD Reporter Assay Kits (Qiagen) according to the manufacturer's instructions. The SMAD reporter is a mixture of an inducible SMAD-responsive luciferase construct and a constitutively expressing Renilla construct.

Colony formation assay. Cell suspensions were seeded into 6-well plates, 300 cells/well. After about 2 weeks, the cells grew to visible colonies and were stained with crystal violet, and then counted using an optical microscope.

Transwell assay. The ability of HCC cells to invade was examined through Matrigel-coated transwell inserts $(8 \mu \mathrm{m}$ pore size, BD Biosciences). In brief, $1 \times 10^{5}$ cells in $200 \mu \mathrm{l}$ FBS-free medium were added in the upper chamber of the Transwell and 10\% FBS containing medium was added 
in the lower chamber. After incubation for 24 hours, cells adhered to the top surface of the membrane were removed with a cotton swab, whereas cells migrated to the bottom surface were fixed with $4 \%$ paraformaldehyde and stained by Giemsa stain. The migrated cells on the bottom surface of the membrane were photographed and counted on an inverted microscope.

Bioluminescence imaging and analysis. $1 \times 10^{6} \mathrm{Huh} 7-\mathrm{luc}$ cells expressing HoxC4 and control vector suspended in $0.1 \mathrm{ml}$ of serum-free DMEM were injected into the flank of each mouse. After 25 days, all mice were anesthetized with ketamine $(100 \mathrm{mg} / \mathrm{kg}$ of body weight $)$ and xylazine $(10 \mathrm{mg} / \mathrm{kg}$ of body weight) before injection. Anesthetized mice were injected with $100 \mathrm{mg} / \mathrm{kg}$ D-Luciferin in PBS. Bioluminescence images were obtained by using the Xenogen IVIS system 5 min after injection. The analysis was performed with LIVINGMAGE software (Xenogen).

Statistical analysis. The unimodal data of HOXC4 expression was dichotomized at the median and Survival analysis was carried out according to the methods of Kaplan and Meier. All calculations were performed with the SPSS18.0 (SPSS Inc., Chicago, IL, USA). Results of in vitro and in vivo experiments were depicted as mean \pm SD. Student's two-sided $\mathrm{t}$-test was used to compare values of test and control samples. A p-value $<0.05$ was considered significant.

A

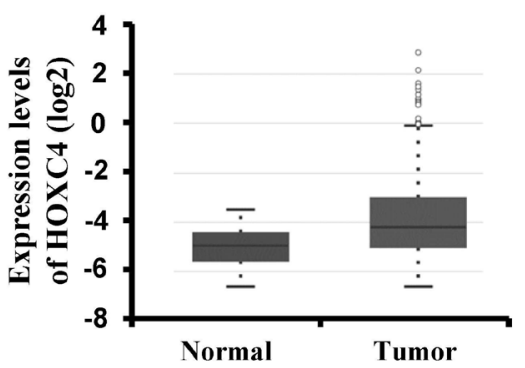

C

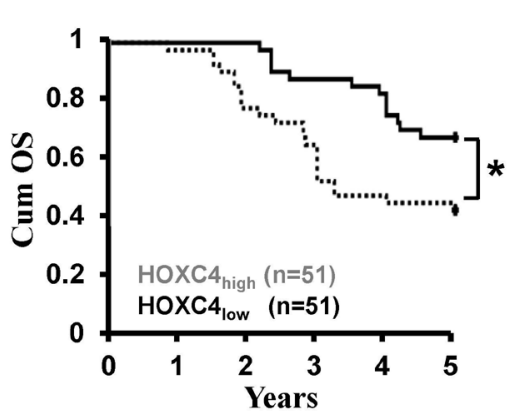

Results

HOXC4 is elevated in HCC and predicts poor outcome. To determine the HOXC4 expression in HCC, we analyzed the expression of HOXC4 in the HCC tissues and the adjacent normal liver tissues from the TCGA database. We observed an increased HOXC4 expression in HCC tissues (Figure 1A). Furthermore, we determined the expression of HOXC4 in 30 cases of HCC and the paired adjacent normal liver tissues by reverse transcription-quantitative PCR (RT-qPCR). The results showed that the expression levels of HOXC4 were significantly upregulated in HCC tissues compared with the adjacent normal liver tissues (Figure 1B). To further investigate the clinical significance of HOXC4 in HCC, we examined the HOXC4 expression in $102 \mathrm{HCC}$ samples by RT-qPCR. The results showed that relative HOXC4 mRNA levels ranged from $2.5 \times 10^{-5}$ to $1 \times 10^{-3}$, with a median level of $6 \times 10^{-4}$. When dichotomized at the median expression level, elevated HOX4 expression was associated with significantly decreased DFS in a Kaplan-Meier analysis (Figure 1C). Furthermore, the number of patients with lung metastasis was higher in the high HOXC4 expression group compared to those with low HOXC4 expression (Figure 1D). Together, these results suggest that HOXC4 is involved in HCC progression.

B

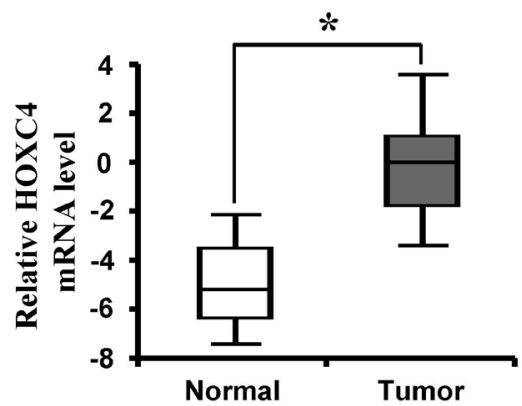

D

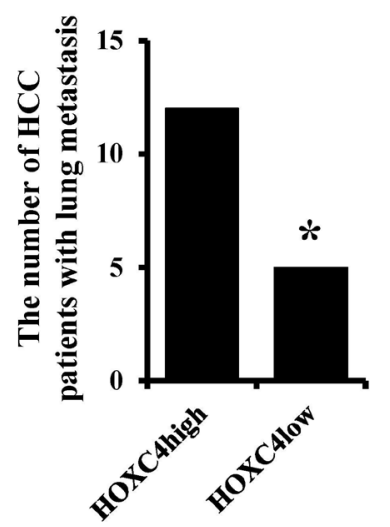

Figure 1. HOXC4 is upregulated in HCC tissues and is associated with shorter overall survival in patients with HCC. A) The relative expression levels of HOXC4 in patients with HCC from the TCGA database. B) The HOXC4 mRNA expression in 30 cases of primary HCC tissues and the paired adjacent normal tissues. C) The Kaplan-Meier analysis of 5-year OS curves stratified by HOXC4 mRNA expression in 102 HCC patients. D) The number of HCC patients with lung metastasis. ${ }^{*} \mathrm{p}<0.05$ 
HOXC4 promotes HCC progression both in vitro and in vivo. To determine the HOXC4 expression in liver cancer cell lines, we detected the HOXC4 expression by western blot. The expression of HOXC4 was high in HepG2 cells (Figure 2A). To evaluate the effect of HOXC4 knockdown on HCC progression, we used two specific siRNAs targeting $\mathrm{HOXC4}$, and both of them could efficiently reduce HOXC4 expression in HepG2 cells (Figure 2B). We examined the effect of HOXC4 knockdown on the invasion of HCC cells. As shown in Figure 2C, the number of migrated cells was much lower in HOXC4-depleted cells compared to that in control cells. Furthermore, the number of colonies was also lower in HOXC4-depleted cells than the control cells by colony formation assay (Figure 2D). For an in-depth understanding of the role of HOXC4 in HCC, we transfected pcDNA3.1HOXC4 into Huh7 cells and constructed the stable cell clone. As shown in Figure 2E, the expression of HOXC4 was signifi- cantly upregulated in HOXC4-transfected cells. The number of migrated cells and colonies was significantly increased in HOXC4-overexpressed Huh7 cells compared to those in control cells by transwell (Figure 2F) and colony formation (Figure 2G) assays, respectively. We next performed xenograft assay in nude mice to determine if HOXC4 overexpression in Huh7 cells was able to affect cancer progression in vivo. As determined by bioluminescence imaging of luciferase activity, Huh7-HOXC4 cells caused a significant metastasis by day 25 after injection (Figure $2 \mathrm{H}$ ). Together, these results indicated that HOXC4 promotes HCC progression both in vitro and in vivo.

HOXC4 induces an EMT-like phenotype by transactivation of Snail. EMT allows epithelial cells to acquire an invasive mesenchymal phenotype and is one of the main mechanisms in cancer metastasis. Next, we determined the expression of EMT-related factors in HOXC4-
A

D
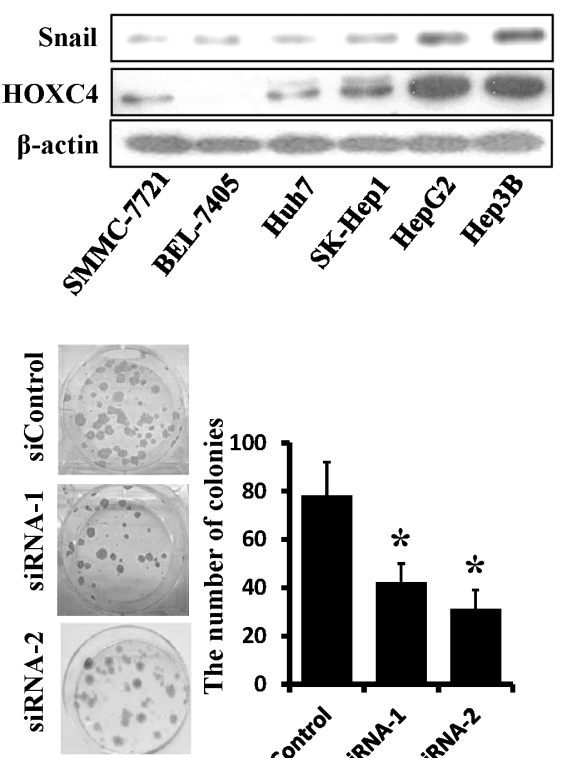

G
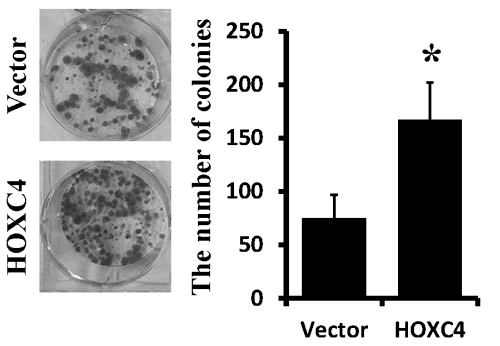

B

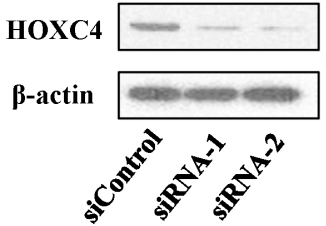

$\mathbf{E}$

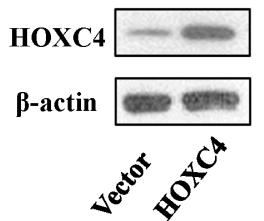

C

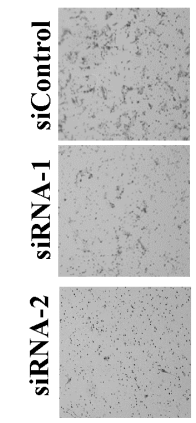

F
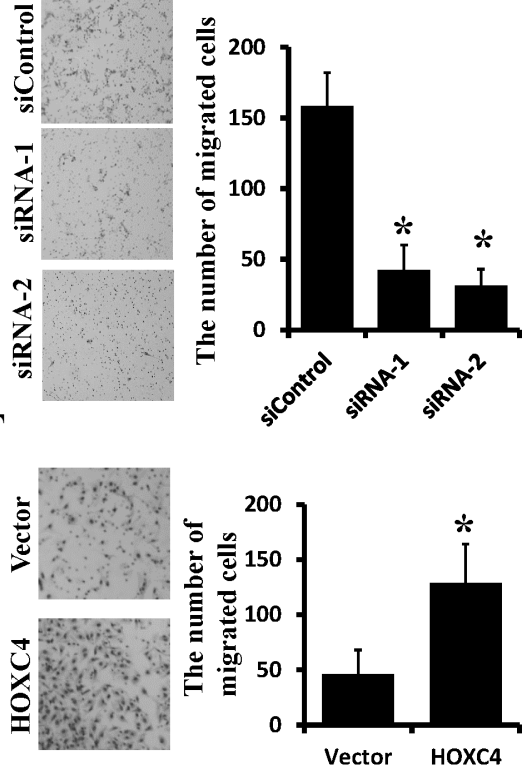

H
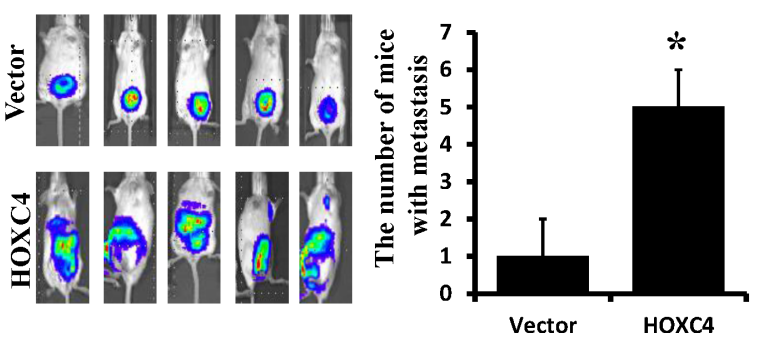

Figure 2. HOXC4 promotes HCC progression both in vitro and in vivo. A) The HOXC4 and Snail expression in liver cancer cell lines determined by western blot. B) The HOXC4 expression in HepG2 cells transfected with siRNAs targeting HOXC4 or siControl determined by western blot. C) Transwell analysis of HOXC4-depleted HepG2 and control cells. D) Colony formation assay of HOXC4-depleted HepG2 and control cells. E) The HOXC4 expression in Huh7 cells stable expressing pcDNA3.1-HOXC4 or vector control determined by western blot. F) Transwell analysis of HOXC4-overexpressed Huh7 and control cells. G) Colony formation assay of HOXC4-overexpressed Huh7 and control cells. H) Representative photos of the general physical condition of xenograft mice carrying Huh7-luc cells expressing either vector control or $\mathrm{HOXC4}$ on 32 days after injection are shown. ${ }^{\star}$ p $<0.05$ 
A

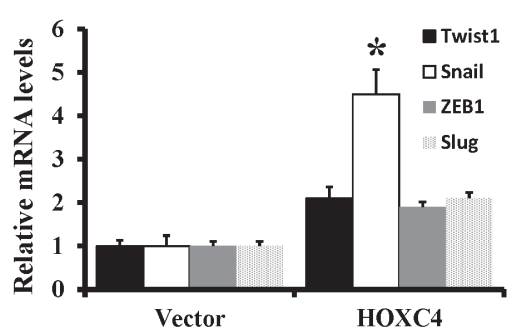

D

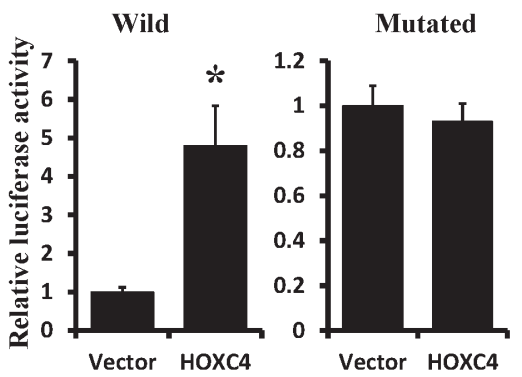

B

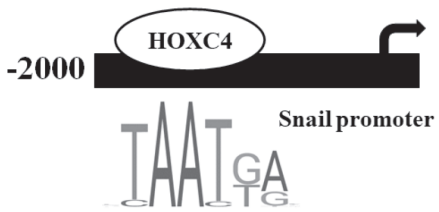

$\mathbf{E}$

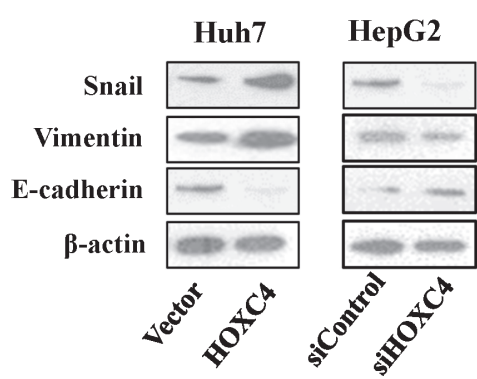

F

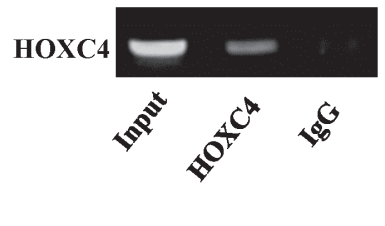

SNAI1 vs HOXC4, 374 samples (LIHC) Data Source starBase v3.0 project

C

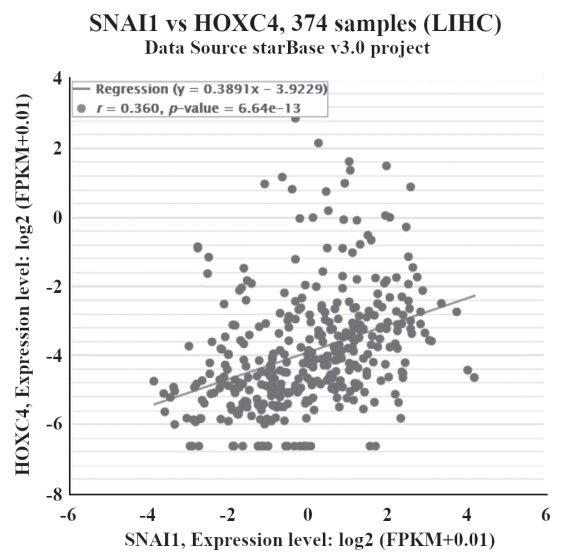

Figure 3. HOXC4 transactivates Snail expression. A) The expression of EMT-related factors in HOXC4-overexpressed Huh7 and control cells determined by RT-qPCR. B) Schematic representation of the 2 kbps upstream of the Snail promoter region. C) Interaction between HOXC4 and the Snail promoter region in HepG2 cells determined by ChIP analysis. D) The wild-type (left) or HOXC4 binding site mutated (TAATGA to AAAAAA; right) Snail promoter activity was measured in HEK-293T cells transfected with pcDNA3.1-HOXC4 or vector by luciferase analysis. E) The expression of EMT markers and Snail in HOXC4-overexpressed Huh7 or HOXC4-depleted HepG2 cells, as well as the control cells determined by western blot. F) The relationship between HOXC4 and Snail expression from the TCGA database. ${ }^{\star} \mathrm{p}<0.05$

overexpressed Huh7 and the control cells by RT-qPCR. As shown in Figure 3A, the expression of Snail was significantly increased in HOXC4-overexpressed Huh7 compared with that in control cells. Furthermore, we found a HOXC4 binding site on the Snail promoter region (Figure 3B). ChIP analysis indicated HOXC4 could bind to the Snail promoter (Figure 3C). To further determine whether Snail is mediated by HOXC4, the promoter region $(-2000$ to +1$)$ was cloned into pGL3-basic luciferase reporter plasmid. As shown in Figure 3D, overexpression of HOXC4 could upregulate the wild-type reporter luciferase activity, whereas mutation of the HOXC4 binding site resulted in complete loss of responsiveness to HOXC4 (Figure 3D). The expression of Snail was also upregulated in HOXC4-overexpressed Huh7 cells shown by western blot (Figure 3E). Furthermore, the results showed that the expression of mesenchymal phenotype cell biomarker Vimentin was increased, whereas epithelial phenotype cell biomarker E-cadherin was decreased in HOXC4-depleted cells (Figure 3E; left). Conversely, depletion of HOXC4 reduced the expression of Snail and Vimentin, whereas the expression of E-cadherin was increased in HepG2 cells (Figure 3E; right). The expression of HOXC4 exhibited a positive relationship with Snail expression in liver cancer cell lines (Figure 2A) and the TCGA database (Figure 3F). Thus, these results indicated that HOXC4 induces an EMT-like phenotype by transactivation of Snail in HCC.
HOXC4 activates TGF- $\beta$ signaling. Transforming growth factor- $\beta$ (TGF- $\beta$ ) is a pleiotropic cytokine, which contributes to wound healing, angiogenesis, fibrosis, and EMT [18]. In addition, a previous study indicated that Snail contributes to cancer development by activation of the TGF- $\beta$ signaling [19]. Thus, we performed luciferase assay to determine the effect of HOXC4 on the TGF- $\beta$ activity. As shown in Figure 4A, the TGF- $\beta$ activity was significantly increased in HOXC4-overexpressed Huh7 cells. In addition, the expression of TGF- $\beta 1$ was upregulated in HOXC4-overexpressed Huh7 cells compared to that in control cells (Figure 4B). To further confirm the TGF- $\beta$ signaling was activated in HOXC4-overexpressed Huh7 cells, we performed immunofluorescence and western blot to examine the localization and expression of Smad2. The results showed that Smad2 translocated into the nucleus in HOXC4-overexpressed Huh7 cells than the control cells (Figure 4C). Furthermore, the expression of phosphorylated Smad2 was greatly increased in HOXC4-overexpressed cells by western (Figure 4D). The number of migrated cells was much lower in HOXC4-overexpressed cells after treatment with TGF- $\beta$ inhibitor SB-431542 (Figure 4E). Furthermore, the TGF- $\beta$ activity was significantly increased in HepG2 cells after TGF- $\beta 1$ treatment, whereas the depletion of HOXC4 could partly abolish this effect (Figure 4F). The number of migrated cells was also lower in HOXC4-depleted 
cells after treatment with TGF- $\beta 1$ (Figure 4G). The KEGG analysis also indicated that HOXC4 is involved in the regulation of TGF- $\beta$ signaling (Supplementary data 1). Together, these results indicated HOXC4 promotes HCC progression through activation of the TGF- $\beta$ signaling.

\section{Discussion}

It is evident that many of the molecular pathways that are involved in carcinogenesis represent aberrations of the normal processes that control embryogenesis. Hox transcrip- tion factors are essential for embryonic development and play critical roles in cell fate determination, differentiation, and proliferation. Aberrations in Hox gene expression have been reported in abnormal development and malignancy, therefore, Hox gene expression could be important in diagnosis and therapy in malignancy [20,21]. Overexpression of Hox genes can transform mammalian cells in vitro and in vivo, and increased Hox gene expression has been detected in many cancer types $[9,22,23]$. Normal Hox gene expression is disrupted and affects various pathways that promote tumorigenesis and metastasis.
A

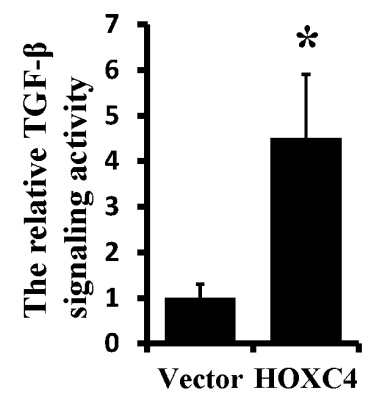

D

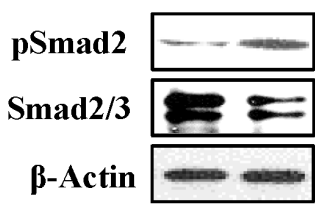<smiles>[C-]1[CH]C=[Ge]=C1</smiles>

F

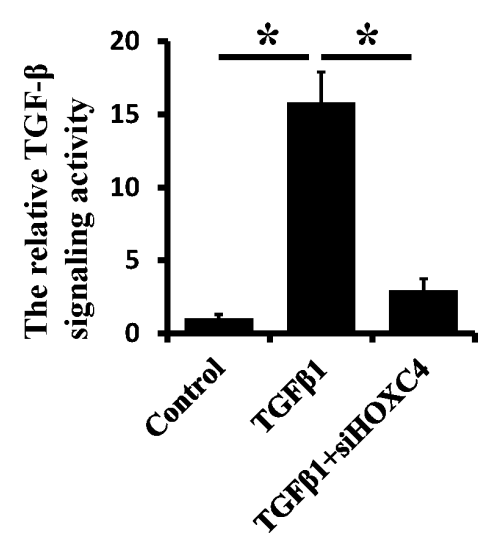

B

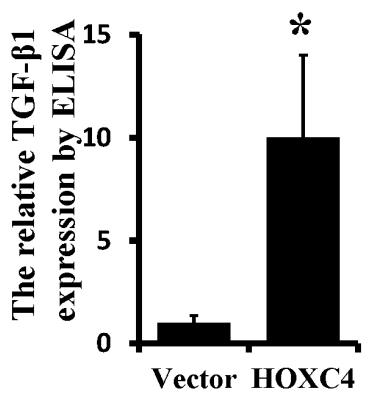

E

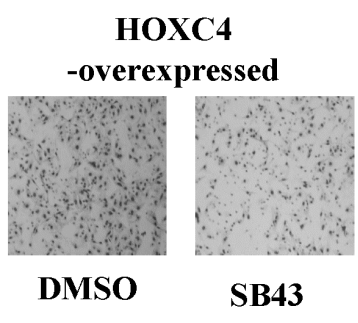

G

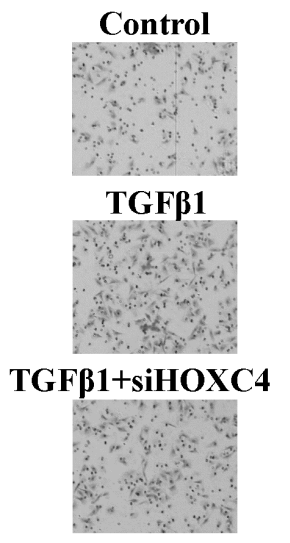

C
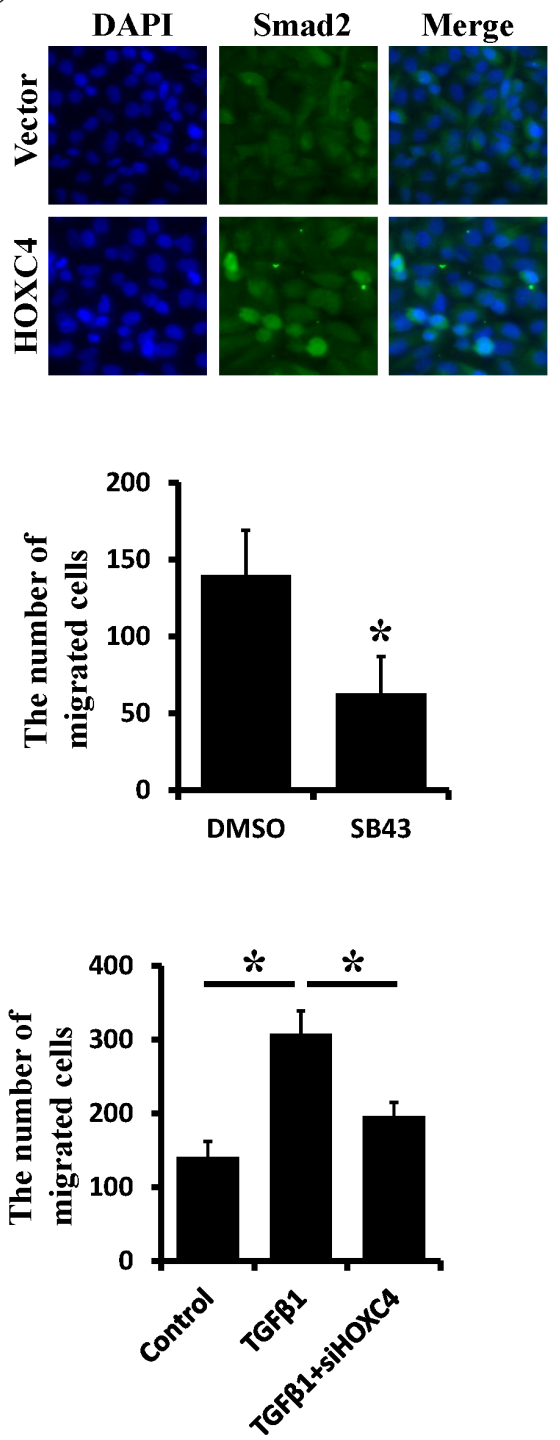

Figure 4. HOXC4 activates TGF- $\beta$ signaling. A) Luciferase reporter analysis of TGF- $\beta$ signaling activity in HOXC4-overexpressed Huh7 and control cells. B) TGF- $\beta 1$ expression in HOXC4-overexpressed Huh7 and control cells by ELISA assay. C) Localization of Smad2 in HOXC4-overexpressed Huh7 and control cells by immunofluorescence assay. D) The expression of Smad 2 and phospho-Smad2 in HOXC4-overexpressed Huh7 and control cells. E) Transwell analysis of Huh7-HOXC4 cells after treatment with TGF- $\beta$ inhibitor SB-431542 or DMSO. F) Luciferase reporter analysis of TGF- $\beta$ signaling activity in HOXC4-depleted HepG 2 and control cells with or without TGF- $\beta 1$ treatment. G) Transwell analysis of HOXC4-depleted HepG 2 cells and control cells with or without TGF- $\beta 1$ treatment. ${ }^{*} \mathrm{p}<0.05$ 
HOXC4 is a HOX family member that presumably functions by binding directly to DNA promoter elements via its homeodomain, which is a DNA binding domain located within its $\mathrm{N}$ terminus [24]. Aberrant expression of HOXC4 is commonly observed in prostate cancer and HOXC4 interacts with HOXB13, FOXA1, and AR, and contributes to the development and progression in prostate cancer [25]. In addition, the expression of HOXC4 is upregulated in melanoma with distant metastasis, and the overexpression of HOXC4 promotes the uveal melanoma development and progression $[12,13]$. In the present study, we demonstrated that HOXC4 is upregulated in the HCC tissues and contributes to HCC progression both in vitro and in vivo, suggesting that HOXC4 functions as an oncogene in HCC.

EMT occurs frequently during normal development and tumorigenesis. EMT is also crucial for the loss of cell polarity of epithelial cells, thus facilitating migratory and invasive behavior during cancer progression $[26,27]$. Our results indicated that HOXC4 induces an EMT-like phenotype in human HCC cells, including downregulation of epithelial marker E-cadherin and upregulation of mesenchymal marker Vimentin. Furthermore, we observed that HOXC4 elevates the expression of EMT-related transcription factor Snail. These findings demonstrated that HOXC4 promotes HCC progression through the induction of EMT. Snail, a zinc-finger transcription factor, plays an important role in the regulation of cell differentiation fate and determination and is also a master EMT regulator in many types of human cancers, including HCC $[28,29]$. Here, we demonstrated that Snail is a target of HOXC4, suggesting that HOXC4 contributes to HCC progression and an EMT-like phenotype by transactivation of Snail.

EMT can be regulated by various growth and differentiation factors, including growth factors that act through receptor tyrosine kinases, such as fibroblast growth factor, TGF- $\beta$, Wnt, and Notch proteins $[30,31]$. Among these, TGF- $\beta$ has received much attention as a major inducer of EMT during fibrosis, embryogenesis, and cancer progression. Moreover, Snail has been widely reported to be involved in the regulation of the TGF- $\beta$ signaling during HCC development and progression [32]. Our results showed that HOXC4 can activate TGF- $\beta$ signaling. Moreover, SB-431542, a TGF- $\beta$ inhibitor, reverses the HOXC4-induced HCC progression, suggesting that HOXC4 promotes HCC progression through activation of TGF- $\beta$ signaling.

In summary, we demonstrated that HOXC4 is upregulated in human HCC and HOXC4 exhibits oncogene activity that promotes the HCC progression both in vitro and in vivo through activation of the TGF- $\beta$ signaling. Furthermore, HOXC4 induces an EMT-like phenotype by transactivating Snail expression. This study suggests that HOXC4 may be a potential target for HCC therapy.

Supplementary information is available in the online version of the paper.
Acknowledgments: This study was supported by the Key Technology R\&D Program of Hebei Province (No. 16277761D).

\section{References}

[1] SIEGEL R, MA J, ZOU Z, JEMAL A. Cancer statistics, 2014. CA Cancer J Clin 2014; 64: 9-29. https://doi.org/10.3322/ caac. 21208

[2] STEIN S, FRITSCH R, LEMAIRE L, KESSEL M. Checklist: vertebrate homeobox genes. Mech Dev 1996; 55: 91-108. https://doi.org/10.1016/0925-4773(95)00494-7

[3] VERAKSA A, DEL CAMPO M, MCGINNIS W. Developmental patterning genes and their conserved functions: from model organisms to humans. Mol Genet Metab 2000; 69: 85-100. https://doi.org/10.1006/mgme.2000.2963

[4] ABATE-SHEN C. Deregulated homeobox gene expression in cancer: cause or consequence? Nat Rev Cancer 2002; 2: 777-785. https://doi.org/10.1038/nrc907

[5] LI B, HUANG Q, WEI GH. The Role of HOX Transcription Factors in Cancer Predisposition and Progression. Cancers (Basel) 2019; 11: 528. https://doi.org/10.3390/cancers11040528

[6] YAN T, OOI WF, QAMRA A, CHEUNG A, MA D et al. HoxC5 and miR-615-3p target newly evolved genomic regions to repress hTERT and inhibit tumorigenesis. Nat Commun 2018; 9: 100. https://doi.org/10.1038/s41467-01702601-1

[7] MILLER GJ, MILLER HL, VAN BOKHOVEN A, LAMBERT JR, WERAHERA PN et al. Aberrant HOXC expression accompanies the malignant phenotype in human prostate. Cancer Res 2003; 63: 5879-5888.

[8] ZHANG X, LI H, ZHEN T, DONG Y, PEI X et al. Shi, hsa_circ_001653 Implicates in the Development of Pancreatic Ductal Adenocarcinoma by Regulating MicroRNA-377Mediated HOXC6 Axis. Mol Ther Nucleic Acids 2020; 20: 252-264. https://doi.org/10.1016/j.omtn.2019.12.028

[9] ZHANG F, REN CC, LIU L, CHEN YN, YANG L et al. Zhang, HOXC6 gene silencing inhibits epithelial-mesenchymal transition and cell viability through the TGF-beta/ smad signaling pathway in cervical carcinoma cells. Cancer Cell Int 2018; 18: 204. https://doi.org/10.1186/s12935-0180680-2

[10] LIU H, ZHANG M, XU S, ZHANG J, ZOU J et al. HOXC8 promotes proliferation and migration through transcriptional up-regulation of TGFbeta1 in non-small cell lung cancer. Oncogenesis 2018; 7: 1. https://doi.org/10.1038/s41389-0170016-4

[11] KIM DY, CHOI SJ, KIM SH, CHUNG HY, YI S et al. Upregulated hoxC4 induces $\mathrm{CD} 14$ expression during the differentiation of acute promyelocytic leukemia cells. Leuk Lymphoma 2005; 46: 1061-1066. https://doi. org/10.1080/10428190500102589

[12] MAEDA K, HAMADA J, TAKAHASHI Y, TADA M, YAMAMOTO Y et al. Altered expressions of HOX genes in human cutaneous malignant melanoma. Int J Cancer 2005; 114: 436-441. https://doi.org/10.1002/ijc.20706 
[13] WU S, CHEN H, ZUO L, JIANG H, YAN H. Suppression of Long non-coding RNA MALAT1 inhibits the development of uveal melanoma via microRNA-608-mediated inhibition of HOXC4. Am J Physiol Cell Physiol 2020; 318: C903-C912. https://doi.org/10.1152/ajpcell.00262.2019

[14] CRITELli R, MilOSA F, FAillaCi F, CONDELlO R, TUROLA E et al. Microenvironment inflammatory infiltrate drives growth speed and outcome of hepatocellular carcinoma: a prospective clinical study. Cell Death Dis 2017; 8: e3017. https://doi.org/10.1038/cddis.2017.395

[15] GURZU S, KOBORI L, FODOR D, JUNG I. Epithelial Mesenchymal and Endothelial Mesenchymal Transitions in Hepatocellular Carcinoma: A Review. Biomed Res Int 2019; 2019: 2962580. https://doi.org/10.1155/2019/2962580

[16] TSUBAKIHARA Y, MOUSTAKAS A. Epithelial-Mesenchymal Transition and Metastasis under the Control of Transforming Growth Factor beta. Int J Mol Sci 2018; 19: 3672. https://doi.org/10.3390/ijms19113672

[17] CANTElli G., CROSAS-MOLIST E., GEORGOUli M., SANZ-MORENO V. TGFBeta-induced transcription in cancer. Semin Cancer Biol 2017; 42: 60-69. https://doi. org/10.1016/j.semcancer.2016.08.009

[18] PRUD'HOMME GJ. Pathobiology of transforming growth factor beta in cancer, fibrosis and immunologic disease, and therapeutic considerations. Lab Invest 2007; 87: 1077-1091. https://doi.org/10.1038/labinvest.3700669

[19] SHIELDS MA, EBINE K, SAHAI V, KUMAR K, SIDDIQUI $\mathrm{K}$ et al. Snail cooperates with KrasG12D to promote pancreatic fibrosis. Mol Cancer Res 2013; 11: 1078-1087. https:// doi.org/10.1158/1541-7786.MCR-12-0637

[20] SHAH N, SUKUMAR S. The Hox genes and their roles in oncogenesis. Nat Rev Cancer 2010; 10: 361-371. https://doi. org/10.1038/nrc2826

[21] SHAH M, CARDENAS R, WANG B, PERSSON J, MONGAN NP et al. HOXC8 regulates self-renewal, differentiation and transformation of breast cancer stem cells. Mol Cancer 2017; 16: 38. https://doi.org/10.1186/s12943-017-0605-z

[22] WANG Y, WANG C, LIU N, HOU J, XIAO W et al. HOXC6 promotes cervical cancer progression via regulation of Bcl2. FASEB J 2019; 33: 3901-3911. https://doi.org/10.1096/ f. 201801099RR
[23] SHEN LY, ZHOU T, DU YB, SHI Q, CHEN KN. Targeting HOX/PBX dimer formation as a potential therapeutic option in esophageal squamous cell carcinoma. Cancer Sci 2019; 110: 1735-1745. https://doi.org/10.1111/cas.13993

[24] SVINGEN T, TONISSEN KF. Hox transcription factors and their elusive mammalian gene targets. Heredity (Edinb) 2006; 97: 88-96. https://doi.org/10.1038/sj.hdy.6800847.

[25] LUO Z, FARNHAM PJ. Genome-wide analysis of HOXC4 and HOXC6 regulated genes and binding sites in prostate cancer cells. PLoS One 2020; 15: e0228590. https://doi. org/10.1371/journal.pone.0228590

[26] DERYNCK R, WEINBERG RA. EMT and Cancer: More Than Meets the Eye. Dev Cell 2019; 49: 313-316. https://doi. org/10.1016/j.devcel.2019.04.026

[27] AIELLO NM, KANG Y. Context-dependent EMT programs in cancer metastasis. J Exp Med 2019; 216: 1016-1026. https://doi.org/10.1084/jem.20181827

[28] CHEN S, WANG G, TAO K, CAI K, WU K et al. LncRNA MALAT1 cooperates with EZH2 to promote hepatocellular carcinoma development by modulating the miR-22/ SNAI1 axis. Cancer Sci 2020; 111: 1582-1595. https://doi. org/10.1111/cas.14372

[29] KONG P, XU E, BI Y, XU X, LIU X et al. Novel ESCC-related gene ZNF750 as potential Prognostic biomarker and inhibits Epithelial-Mesenchymal Transition through directly depressing SNAI1 promoter in ESCC. Theranostics 2020; 10: 1798-1813. https://doi.org/10.7150/thno.38210

[30] XU J, LAMOUILLE S, DERYNCK R. TGF-beta-induced epithelial to mesenchymal transition. Cell Res 2009; 19: 156172. https://doi.org/10.1038/cr.2009.5

[31] MOUSTAKAS A, HELDIN CH. Signaling networks guiding epithelial-mesenchymal transitions during embryogenesis and cancer progression. Cancer Sci 2007; 98: 1512-1520. https://doi.org/10.1111/j.1349-7006.2007.00550.x

[32] BELLOMO C, CAJA L, FABREGAT I, MIKULITS W, KARDASSIS D et al. Snail mediates crosstalk between TGFbeta and LXRalpha in hepatocellular carcinoma. Cell Death Differ 2018; 25: 885-903. https://doi.org/10.1038/s41418-0170021-3 